\title{
Creating Novel Structures in Food Materials: The Role of Well-Defined Shear Flow
}

\author{
A. J. van der Goot $\cdot$ S. H. Peighambardoust • \\ C. Akkermans • J. M. van Oosten-Manski
}

Received: 28 November 2007 / Accepted: 5 March 2008/Published online: 2 April 2008

(C) The Author(s) 2008

\begin{abstract}
Structure formation in food materials is influenced by the ingredient properties and processing conditions. Until now, small structural elements, such as fibrils and crystals, have been formed using self-assembly, while processing was applied to create relatively large structures. The effect of self-assembly under flow is rarely studied for food materials, but it is widely studied for non-food systems. The use of well-defined flow, often simple shear, turned out to be essential to study and control the structure formation process in foods as well. This observation encouraged us to develop a number of different shearing devices that allowed processing of biopolymer systems under simple shear flow. This paper reviews our main findings. In the case of protein fibrillization, the shear rate was found to control the growth rate as well as the properties of the fibrils formed. In the case of dough processing, simple shear flow made the product more process tolerant and induced gluten migration. The use of shear flow for dense caseinate dispersions led to hierarchically structured and fibrous material. Based on the presented results, we conclude that introducing simple shear flow in food structuring processes can lead to a much
\end{abstract}

A. J. van der Goot $(\bowtie) \cdot$ C. Akkermans

Food Engineering Group, Wageningen University,

P.O. Box 8129, 6700 EV Wageningen, The Netherlands

e-mail: Atzejan.vandergoot@wur.nl

S. H. Peighambardoust

Food Science and Technology Group, Faculty of Agriculture, University of Tabriz,

Tabriz 5166614766, Islamic Republic of Iran

J. M. van Oosten-Manski

Fonterra,

Private Bag 11029, Dairy Farm Road,

Palmerston North, New Zealand broader range of structures, thereby better utilizing the full potential of food ingredients.

Keywords Shear flow · Structure formation · Biopolymers

\section{Introduction}

Structures in food or food-related materials are formed through self-assembly or forced assembly. Self-assembly is the spontaneous formation of small structural elements such as aggregates, fibrils or micelles through molecular attractive properties. Forced assembly leads to the creation of large structures with their formation being fully controlled. However, a size range exists, e.g., colloidal domain structures $(10 \mathrm{~nm}-10 \mathrm{um})$, where structure formation is strongly influenced by both self assembly and superimposed process conditions. Until now, most structuring processes such as extrusion or spinning do not make extensive use of the self-assembly potential of biopolymers. As a result, the products obtained are not structured on a molecular and microscopic scale. For example, it was reported that soy proteins remain globular and randomly ordered on a microscopic scale after spinning, while the product obtained was macroscopically anisotropic. ${ }^{1}$ The lack of structuring molecular of microscopic scale can be explained by the fact that the flow inside the equipment used is not well-defined and varies with the actual position inside the equipment. ${ }^{2,3}$

When looking at studies in which model systems are studied, researchers often make use of simple shear flow. It allows them to make a broad range of structures for a given composition. ${ }^{4-7}$ In addition, the structure formation could be followed by measuring the effect of the shear rate on the rheological properties, such as viscosity and normal 
forces. ${ }^{8,9}$ These studies indicated that the structure formation is a result of interactions present in the dispersion in relation to the shear stress applied. The exact nature of the interactions depends on the model system studied. In the case of a colloidal dispersion, long-range electrostatic interactions and short-range Van der Waals interactions affect the structure. For polymer solutions, depletion interactions or entanglements might play an important role. $^{10,11}$

Using this information, we started to develop processing systems, so-called shearing devices, for (concentrated) biopolymer systems. The use of these shearing devices allowed us to create a wide range of interesting product structures. The shearing devices are unique in the combination of a simple shear flow profile and large shear stresses. Secondary flows such as Taylor flows were suppressed by using sufficiently low shear rates. In this paper, we summarize our main findings in three different applications: (1) protein fibrillization, (2) dough systems, and (3) dense caseinate systems.

\section{The Role of Simple Shear Flow in Protein Fibrillization}

Various protein solutions ( $\beta$-lactoglobulin, ${ }^{12}$ lysosyme, ${ }^{13}$ ovalbumine, ${ }^{14}$ soy ${ }^{15}$ ) can form fibrils when they are heated at low $\mathrm{pH}$, using a low to moderate protein concentration $(0.5-7 \mathrm{wt} \%)$. In the case of $\beta$-lactoglobulin, the fibrils formed have a typical thickness of $\sim 4 \mathrm{~nm}^{16}$ and a length between 1 and $10 \mu \mathrm{m}$ (Figure 1). The fibrils are formed through a nucleation and growth mechanism. The formation of the nucleus of a fibril will predominantly be controlled through self-assembly because of the small size of this nucleus. However, once a fibril is formed, flow could influence the growth process.

Akkermans et al. ${ }^{17}$ studied the effect of simple shear flow on the fibril growth of whey protein isolate (contain-

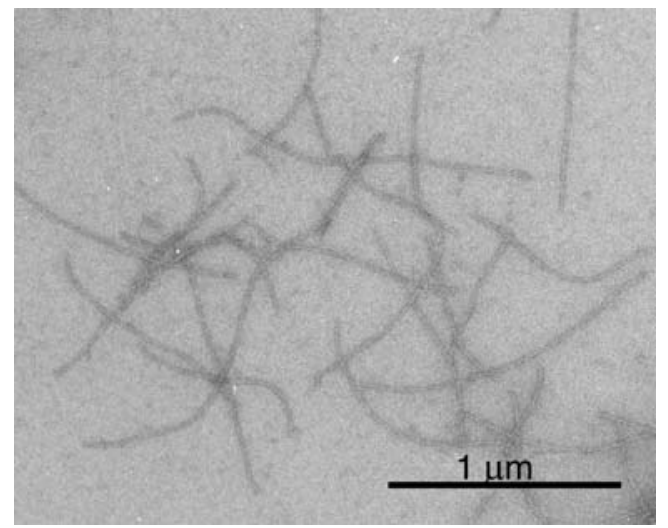

Fig. 1 Transmission electron microscopy micrograph of fibrils obtained after heating a $5.2 \mathrm{wt} \%$ whey protein isolate solution at $90{ }^{\circ} \mathrm{C}$ for $2 \mathrm{~h}$ at $\mathrm{pH} 2$ using a shear rate of $337 \mathrm{~s}^{-1}$

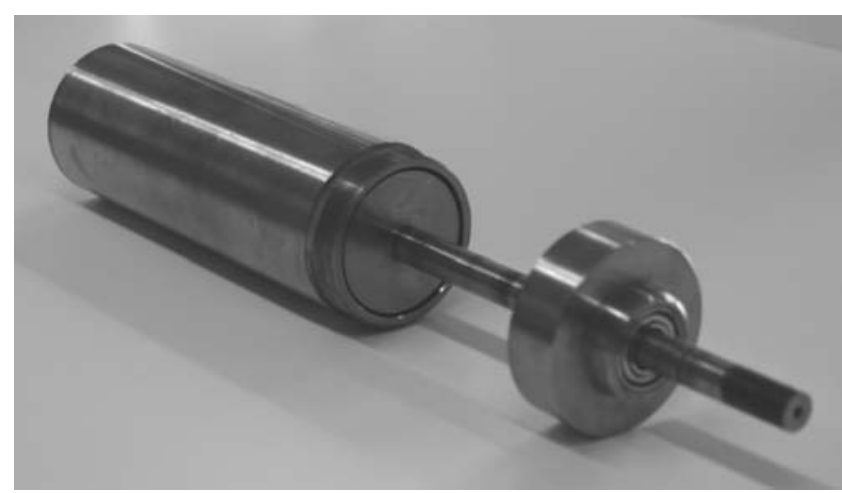

Fig. 2 Shearing device with rotating inner cylinder (diameter $40 \mathrm{~mm}$ ) and static outer cylinder (diameter $42 \mathrm{~mm}$ )

ing $\sim 70 \% \beta$-lactoglobulin). Figure 2 shows the shearing device that was used during this study. It was derived theoretically (based on the tumbling motion a fibril makes in the flow field of simple shear) that the fibril growth rate scales with $\sim \dot{\gamma}^{2 / 3}$ assuming that the growth is limited by the supply of protein monomers. Up to moderate shear rates $\left(\dot{\gamma} \leq 337 \mathrm{~s}^{-1}\right)$, this scaling rule could be applied to the experimental results. At a higher shear rate, the experimentally observed growth rate was lower, probably due to the

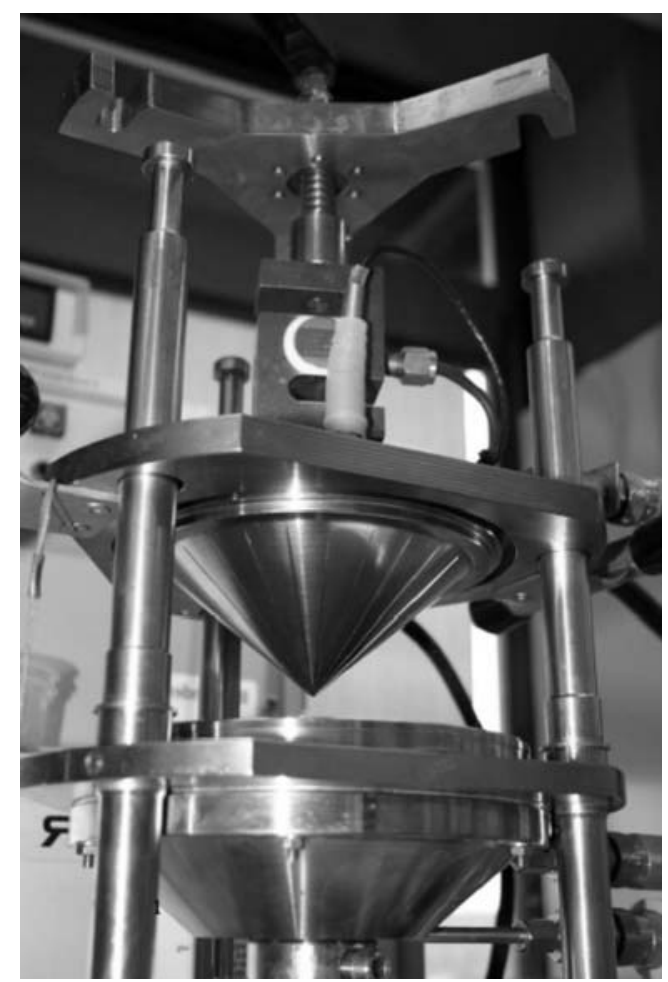

Fig. 3 Shearing device used for processing concentrated biopolymer systems. The diameter of the upper cone is $12 \mathrm{~cm}$. The cone angle is $100^{\circ}$, the angles between the cones are $2.5^{\circ}$. The rotational speed can be varied from 5 to $250 \mathrm{rpm}$, leading to a shear rate ranging from 12 to $600 \mathrm{~s}^{-1}$. The maximum shear stress that can be applied is $40 \mathrm{kPa}$. The system is temperature controlled through an external water bath. More details can be found in Peighambardoust et al. ${ }^{19,23}$ and Manski et al. ${ }^{24}$ 

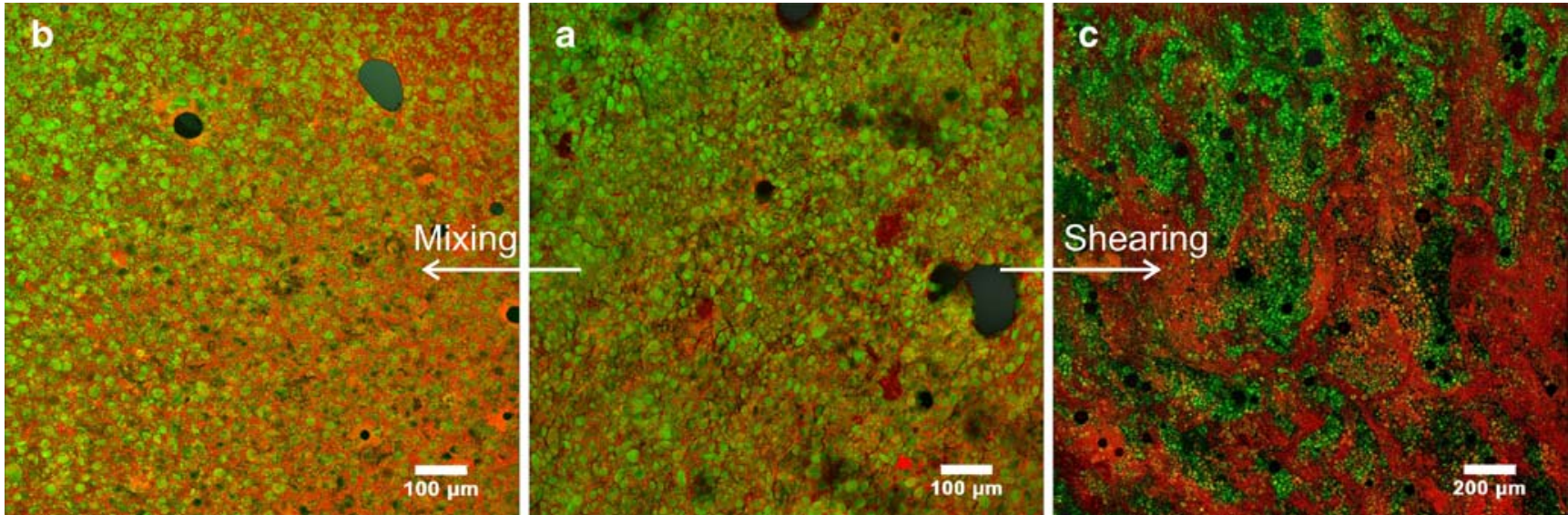

Fig. 4 CSLM pictures of dough microstructures: a Starting material (hydrated dough); b prolonged Z-blade-mixed dough; and $\mathbf{c}$ simple sheared dough. Starch is green, gluten is red, and air bubbles appear as black spots

fact that a high shear rate interferes with the formation of mature fibril bonds. Up to moderate shear rates $\left(\dot{\gamma} \leq 337 \mathrm{~s}^{-1}\right)$, the shear flow also influenced the length of the fibrils: a higher shear rate (up to $600 \mathrm{~s}^{-1}$ ) resulted in shorter fibrils. From this work, it was concluded that the shear rate can be used to control the total fibril length concentration and length of the fibrils formed.

\section{The Role of Simple Shear Flow in Dough Systems}

Wheat dough is made by combining flour and water upon mixing. When mixing dough, the gluten phase forms a continuous network throughout the dough. This network formation can be interpreted as a local phase separation of starch and gluten, which is governed by the ability of gluten to aggregate and the dilatant behavior of starch. ${ }^{18}$ The typical size of a gluten domain remains rather small upon mixing. Prolonged mixing disrupts the gluten network, resulting in a homogeneous dispersion (Figure 4).

Peighambardoust et al. ${ }^{19-21}$ studied the effect of simple shear flow on dough using an in-house developed shearing device (Figure 3). The design of this device was based on a cone-in-cone system. This device enabled us to process dough using a comparable energy input $(100-500 \mathrm{~kJ} / \mathrm{kg})$ as applied during traditional dough mixing in a Z-blade mixer. It turned out that simple shear processing made the gluten process tolerant. The amount of glutenin macro polymer (GMP) that could be extracted from the dough using a $1 \%$ sodium dodecyl sulfate solution remained constant upon shearing. Besides, the sheared dough kept its strainhardening properties, and the dough did not become sticky upon prolonged shear processing. When the dough was mixed in a Z-blade mixer, the amount of GMP decreased sharply upon processing. In addition, the dough lost its strain-hardening properties and became sticky upon pro- longed mixing. Using confocal scanning laser microscopy (CSLM), it was observed that the gluten network formed after short mixing was disrupted completely after longer mixing. In other words, prolonged mixing of dough led to over-processing contrary to shearing.

When the dough structure was analyzed, it was observed that simple shear processing transformed the relatively homogeneous dough into a rather heterogeneous material as a result of local separation of starch particles and gluten (Figure 4). Although most of the gluten patches were slightly anisotropic, there was no clear correlation with flow direction. Structures oriented perpendicular to as well as parallel with the flow direction were obtained. Compared to mixing, gluten could form very large structures. Peressini et al. ${ }^{22}$ showed that the shear rate could be used to control the gluten domain size. At low shear rate $\left(\dot{\gamma}=7.4 \mathrm{~s}^{-1}\right)$, the gluten domains were clearly visible by eye, suggesting that the domains were at least several millimeters in diameter.

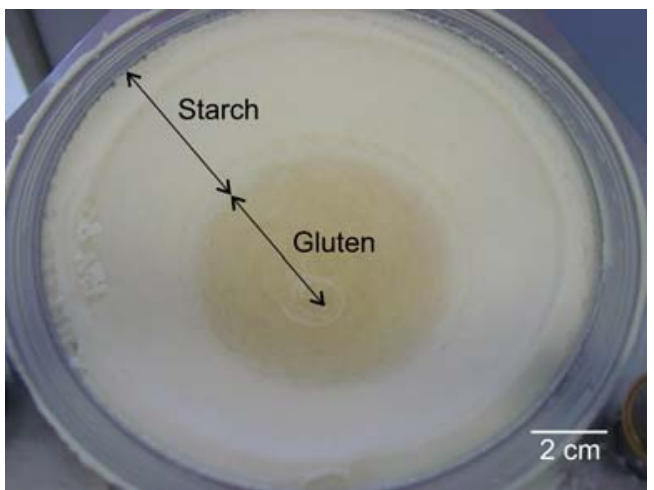

Fig. 5 Separation of dough (dry matter content $56 \mathrm{wt} \%$ ) into gluten and starch. The dough was sheared at a constant shear rate of $24 \mathrm{~s}^{-1}$ for $60 \mathrm{~min}$ at $10{ }^{\circ} \mathrm{C}$ using the shearing device shown in Figure 3 

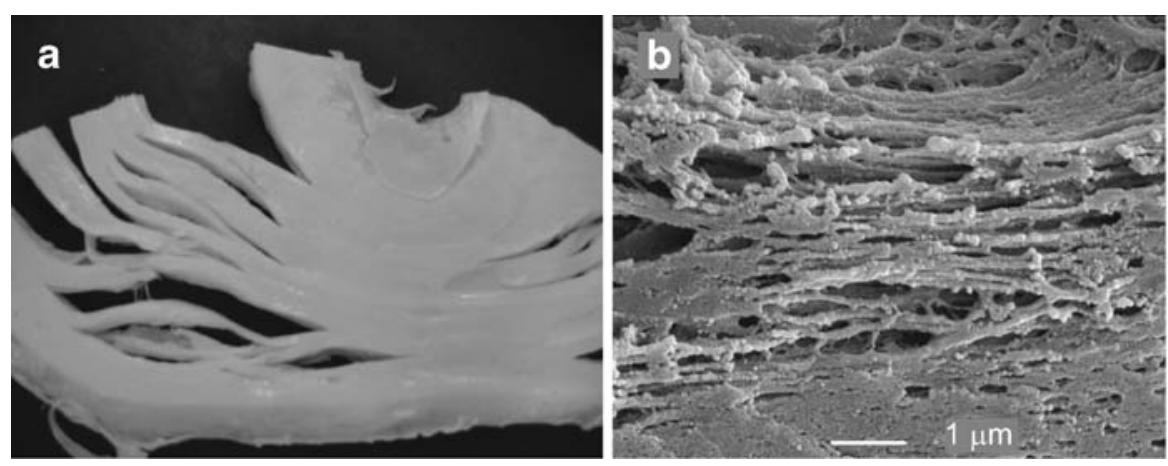

$30 \mathrm{~min}$, and the process temperature was $50{ }^{\circ} \mathrm{C}$. The size of photo $\mathrm{a}$ is about $10 \mathrm{~cm}$. c Microstructure (SEM) of a NaCas dispersion (30 $\mathrm{wt} \%$ in water) including $15 \mathrm{wt} \%$ fat, processed identically as the CaCas dispersion
When shearing dough in a curvilinear shear field, Peighambardoust et al. ${ }^{23}$ showed significant gluten migration towards the apex of the cone. Figure 5 shows the separation of dough into a yellow gluten-rich phase and a white starch-rich phase. A prerequisite for the inward migration was the formation of large gluten domains. The macroscopic migration was explained by considering the elastic nature of the gluten phase, which led to a driving force for the gluten phase to move towards the apex of the cone upon shearing.

The use of simple shear flow in the case of dough leads to structures in dough that could not be obtained using mixing conditions. The fact that simple shear processing does not alter the vitality of gluten (as measured by GMP) combined with the fact that no reorientation hinders the full assembly of gluten structures under flow explains the structure formation process.

\section{The Role of Simple Shear Flow in Dense Caseinate Dispersions}

Calcium caseinate dispersions could be transformed into a wide range of structures with morphologies varying from isotropic and non-fibrous to anisotropic and highly fibrous. $^{24,25}$ Figure 6 shows an example of a fibrous structure that was obtained using the shearing device shown in Figure 3. Figure 6 illustrates that this protein-rich material possesses a hierarchical structure of which the smallest fibrils have a diameter in the order of $100 \mathrm{~nm}$. The use of well-defined shear flow turned out to be essential because the use of a mixer led to an isotropic dispersion of calcium caseinate (CaCas). Comparable experiments with sodium caseinate (NaCas) did not result in fibrous structures either (see Figure $6 \mathrm{c}$ ). ${ }^{26}$
The different behaviors of $\mathrm{NaCas}$ and $\mathrm{CaCas}$ under simple shear flow were explained by considering the internal structure of the dispersions, which was greatly influenced by presence of calcium in the system. The CaCas dispersion contained larger micelles $(\sim 120 \mathrm{~nm})$ than the $\mathrm{NaCas}$ dispersion $(\sim 25 \mathrm{~nm}),{ }^{27}$ which makes the CaCas micelles more susceptible to hydrodynamic (shear) flow. In addition, due to the presence of calcium, it is expected that the attractive forces between the micelles is larger in the case of CaCasdispersion. The dissimilarities led to large differences in rheological behavior as can be observed in Figure 7. The $\mathrm{CaCas}$ dispersion showed shear-thickening at low shear rates, followed by a sudden drop in viscosity, and subsequently shear-thinning at higher shear rates. In contrast, $\mathrm{NaCas}$ showed moderate shear-thinning, liquid-like behavior, suggesting that the internal structure was not significantly altered by shear. Therefore, it seems that the formation of the small fibrils (diameter $\sim 100 \mathrm{~nm}$ ) was caused by a subtle

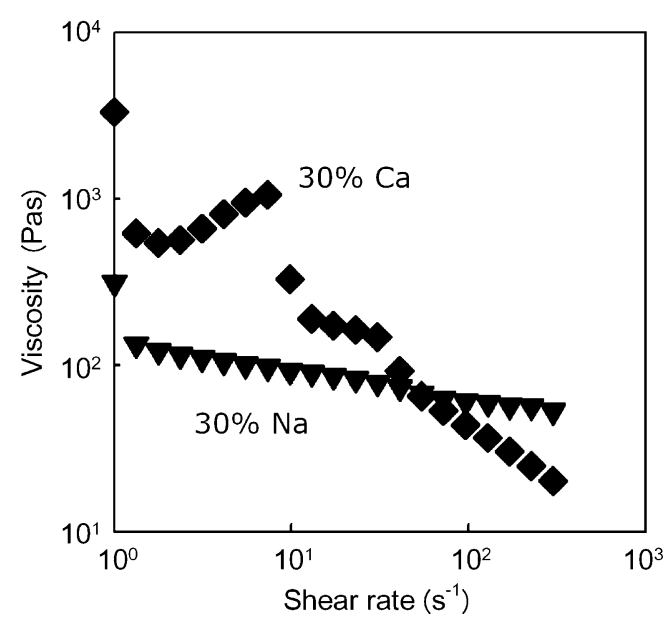

Fig. 7 The effect of shear rate on the viscosity of a $30 \mathrm{wt} \% \mathrm{CaCas}$ and a $30 \mathrm{wt} \% \mathrm{NaCas}$ dispersion 
interplay between dispersion properties (e.g., micelle size, micelle concentration, interaction between the micelles, calcium sensitivity) and the simple shear flow applied. The structure formation could thus be considered as a result of self-assembly under flow. It should be noted however that an additional solidification process was needed to obtain the fibrous solid material. We applied a solidification process based on enzymatic cross-linking using transglutaminase to fixate the aligned micelles into fibrils. Without the solidification, the material became hardly anisotropic. However, too much cross-linking made the material unable to adapt itself to the shear flow applied, which led to fracture of the fibrous structure and syneresis.

The question now remains whether fibril formation can also be obtained in other systems or whether we make use of very specific Cacas-dispersion properties. We think that the latter situation is the case because caseinates (including CaCas) have unique protein properties. Nevertheless, the observations described above resemble the shear-induced structure formation reported for model systems. ${ }^{6,10}$ In those systems, shear flow could be used to create anisotropic structures. The concentration of structural elements, such as micelles, their interaction, and their size were used to explain the behavior of these dispersions under flow.

\section{Concluding Remarks}

Introducing simple shear flow as process parameter is a promising tool for the creation of innovative structures in food products. In biopolymer systems, the use of simple shear flow results in product structures that cannot be obtained when using traditional mixing conditions. This is caused by the fact that simple shear flow allows the biopolymer systems to form structures based on a kind of self-assembly under flow. Parallels between the systems described above and polymer model systems could be further explored to get more insights into the structure-formation process occurring in biopolymer systems.

Open Access This article is distributed under the terms of the Creative Commons Attribution Noncommercial License which permits any noncommercial use, distribution, and reproduction in any medium, provided the original author(s) and source are credited.

\section{References}

1. V. Rampon, R. Robert, N. Nicolas, E. Dufour, Protein structure and network orientation in edible films prepared by spinning process J. Food Sci. 64(2), 313-316 (1999)
2. T.R.G. Jongen, M.V. Bruschke, J.G. Dekker, Analysis of dough kneaders using numerical flow simulations Cereal Chem. 80(4), 383-389 (2003)

3. A.J. Van der Goot, J.M. Manski, in Creation of novel microstructures through processing, ed. by D.J. McClements. Understanding and controlling the microstructure of complex foods (Woodhead Publishing Limited, Abington Hall, Abington, Cambridge, CB1 6AH, England, 2007)

4. A. Onuki, Effects of shear-flow and viscosity difference on phaseseparation Int. J. Thermophys. 16(2), 381-390 (1995)

5. P. Butler, Shear induced structures and transformations in complex fluids Curr. Opin. Colloid Interface Sci. 4(3), 214-221 (1999)

6. J. Vermant, Large-scale structures in sheared colloidal dispersions Curr. Opin. Colloid Interface Sci. 6(5-6), 489-495 (2001)

7. B. Wolf, W.J. Frith, S. Singleton, M. Tassieri, I.T. Norton, Shear behaviour of biopolymer suspensions with spheroidal and cylindrical particles Rheol. Acta. 40(3), 1435-1528 (2001)

8. S. Saito, T. Hashimoto, Critical conditions for structure formation in semidilute polymer solutions induced under continuous shear flow J. Chem. Phys. 114(23), 10531-10543 (2001)

9. J.J. Magda, C.S. Lee, S.J. Muller, R.G. Larson, Rheology, flow instabilities and shear induced diffusiton in polystyrene solutions Macromolecules. 26(7), 1696-1706 (1993)

10. J. Vermant, L. Raynaud, J. Mewis, B. Ernst, G.G. Fuller, Largescale bundle ordering in sterically stabilized latices J. Colloid Interface Sci. 211(2), 221-229 (1999)

11. B. Belzung, F. Lequeux, J. Vermant, J. Mewis, Flow-induced anisotropy in mixtures of associative polymers and latex particles J. Colloid Interface Sci. 224(1), 179-187 (2000)

12. C. Veerman, H. Ruis, L.M.C. Sagis, E. Van der Linden, Effect of electrostatic interactions on the percolation concentration of fibrillar beta-lactoglobulin gels Biomacromolecules. 3, 869-873 (2002)

13. L.N. Arnaudov, R. De Vries, Thermally induced fibrillar aggregation of hen egg white lysozyme Biophys. J. 88, 515-526 (2005)

14. C. Veerman, G. de Schiffart, L.M.C. Sagis, E. van der Linden, Irreversible self-assembly of ovalbumin into fibrils and the resulting network rheology Int. J. Biol. Macromol. 33(1-3), 121-127 (2003)

15. C. Akkermans, A.J. Van der Goot, P. Venema, H. Gruppen, J.M. Vereijken, E. Van der Linden, R.M. Boom, Micrometer-sized fibrillar protein aggregates from soy glycinin and soy protein isolate J. Agric. Food Chem. 55(24), 9877-9882 (2007)

16. L.N. Arnaudov, R. de Vries, H. Ippel, C.P.M. Van Mierlo, Multiple steps during the formation of beta-lactoglobulin fibrils Biomacromolecules. 4, 1614-1622 (2003)

17. C. Akkermans, A.J. Van der Goot, P. Venema, E. Van der Linden, R.M. Boom, Formation of fibrillar whey protein aggregates: influence of heat- and shear treatment and resulting rheology. Food Hydrocoll. (2008 in press). DOI 10.1016/j.foodhyd. 2007.07.001

18. R. Kieffer, N. Stein, Demixing in wheat doughs - its influence on dough and gluten rheology Cereal Chem. 76(5), 688-693 (1999)

19. S.H. Peighambardoust, A.J. van der Goot, R.J. Hamer, R.M. Boom, A new method to study simple shear processing of wheat gluten-starch mixtures Cereal Chem. 81(6), 714-721 (2004)

20. S.H. Peighambardoust, A.J. van der Goot, T. van Vliet, R.J. Hamer, R.M. Boom, Microstructure formation and rheological behaviour of dough under simple shear flow J. Cereal Sci. 43(2), 183-197 (2006)

21. S.H. Peighambardoust, S. van Brenk, A.J. van der Goot, R.J. Hamer, R.M. Boom, Dough processing in a Couette-type device with varying eccentricity: Effect on glutenin macro-polymer properties and dough micro-structure J. Cereal Sci. 45(1), 34-48 (2007) 
22. D. Peressini, S.H. Peighambardoust, R.J. Hamer, A. Sensidoni, A. J. van der Goot, Effect of shear rate on the microstructure and rheological properties of sheared wheat doughs. J Cereal Sci. (2008 in press) DOI 10.1016/j.jcs.2007.10.008

23. S.H. Peighambardoust, R.J. Hamer, R.M. Boom, A.J. Van der Goot, Migration of gluten under shear flow as a novel mechanism for separating wheat flour into gluten and starch. J Cereal Sci. (2008 in press) DOI 10.1016/j.jcs.2007.10.005

24. J.M. Manski, A.J. van der Goot, R.M. Boom, Formation of fibrous materials from dense calcium caseinate dispersions Biomacromolecules. 8(4), 1271-1279 (2007)
25. J.M. Manski, E.E.J. Van der Zalm, A.J. Van der Goot, R.M. Boom, Influence of process parameters on formation of fibrous materials from dense calcium caseinate dispersions Food Hydrocoll. 22(4), 587-600 (2008)

26. J.M. Manski, L.E. Van Riemsdijk, A.J. Van der Goot, R.M. Boom, Importance of intrinsic properties of dense caseinate dispersions for structure formation Biomacromolecules. 8(11), 3540-3547 (2007)

27. W.Y. Aalbersberg, R.J. Hamer, P. Jasperse, H.H.J. De Jongh, C.G. De Kruif, P. Walstra, F.A. De Wolf, Industrial proteins in perspective. 1 ed. vol. 23 (Elsevier Science, Amsterdam, 2003) 